\title{
Dexamethasone treatment does not inhibit fibroproliferation in chronic lung disease of prematurity
}

\author{
W.A. Dik*, M.A. Versnel*, B.A. Naber*,\#, D.J. Janssen" ${ }^{\#}$, A.H. van Kaam", L.J.I. Zimmermann ${ }^{\#}$
}

Dexamethasone treatment does not inhibit fibroproliferation in chronic lung disease of prematurity. W.A. Dik, M.A. Versnel, B.A. Naber, D.J. Janssen, A.H. van Kaam, L.J.I. Zimmermann. (C) ERS Journals Ltd 2003.

ABSTRACT: Pulmonary fibrosis results from excessive fibroblast proliferation and increased collagen deposition and occurs in chronic lung disease of prematurity (CLD). Platelet-derived growth factor (PDGF)-BB is mitogenic for fibroblasts and levels are increased in fibrotic lung disorders. Systemic dexamethasone (DEX) treatment improves pulmonary function and reduces inflammation in infants with or at risk of CLD. However, the effect of DEX treatment on fibroblast activity, PDGF-BB and collagen synthesis in the lungs of CLD patients is uncertain.

Bronchoalveolar lavage (BAL) fluids, obtained from 15 infants at risk of CLD before and after DEX treatment, were analysed for fibroblast mitogenicity, PDGF-BB,

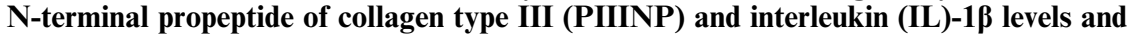
inflammatory cell numbers.

After DEX treatment, the mitogenic activity of BAL fluid for fibroblasts was not reduced but increased. The change in mitogenicity correlated with a change in BAL fluid PDGF-BB levels. Furthermore, BAL fluid-induced fibroblast proliferation was blocked using an inhibitor of the PDGF receptor. DEX treatment did not influence PIIINP levels, but reduced IL-1 $\beta$ levels and inflammatory cell numbers in BAL fluid.

This study suggests that dexamethasone treatment does not reduce fibroblast proliferation despite apparent downregulation of inflammation. The present findings do not support the use of dexamethasone for prevention of the fibrotic response in infants at risk of chronic lung disease of prematurity.

Eur Respir J 2003; 21: 842-847.
Depts of *Immunology and ${ }^{\#}$ Paediatrics, division of Neonatology, Erasmus Medical Centre, Rotterdam, and Neonatology, Emma Children's Hospital, Academic Medical Centre, University of Amsterdam, Amsterdam, the Netherlands.

\author{
Correspondence: W.A. Dik \\ Dept of Immunology \\ Erasmus MC \\ P.O. Box 1738 \\ 3000 DR Rotterdam \\ The Netherlands \\ Fax: 31104089456 \\ E-mail: w.dik@erasmusmc.nl
}

Keywords: Bronchoalveolar lavage chronic lung disease of prematurity

dexamethasone

fibroblast proliferation

platelet-derived growth factor

Received: August 12002

Accepted after revision: December 102002

This study was supported by a grant from the Sophia Foundation for Medical Research, Rotterdam, the Netherlands.
Neonatal respiratory distress syndrome (RDS) is characterised by pulmonary inflammation with neutrophils and macrophages as the main cell types $[1,2]$. RDS can progress towards chronic lung disease (CLD) of prematurity, also known as bronchopulmonary dysplasia. Risk factors for the development of CLD include premature birth with concomitant lung immaturity, mechanical ventilator-induced lung injury, oxygen toxicity and pulmonary inflammation [3].

Pulmonary fibrosis is characterised by excessive fibroblast proliferation and increased collagen deposition [4] and is a common feature in infants dying because of CLD [5]. Increased pulmonary levels of fibrogenic mediators, such as fibronectin and transforming growth factor- $\beta_{1}$, which increase proliferation and collagen synthesis by fibroblasts, have been associated with CLD development $[6,7]$.

Treatment with systemic dexamethasone (DEX) is commonly used in infants with or at risk of CLD and improves pulmonary function, facilitates weaning from the ventilator and reduces pulmonary inflammation [8]. However, it has been suggested that DEX treatment may not inhibit the development of pulmonary fibrosis in CLD, since it does not decrease fibronectin concentrations in bronchoalveolar lavage (BAL) fluid [6]. Conversely, DEX treatment of CLD infants results in decreased urinary excretion of hydroxyproline, indicating suppressed collagen synthesis in these infants [9]. However, the specific effect of DEX treatment on lung collagen synthesis during CLD development remains unclear, as urinary excretion of hydroxyproline reflects total body collagen synthesis.

BAL fluid from adults with acute respiratory distress syndrome (ARDS), who are at risk of pulmonary fibrosis, is mitogenic for lung fibroblasts in vitro, indicating the presence of soluble mitogens [10]. Platelet-derived growth factor (PDGF)-BB is a potent mitogen for fibroblasts and increased pulmonary levels are associated with pulmonary fibrosis [4]. Additionally, PDGF-BB has been shown to contribute to BAL fluid-induced fibroblast proliferation in an animal model of bleomycininduced acute lung injury [11]. It has been reported that expression of PDGF-B messenger ribonucleic acid (mRNA) by alveolar macrophages, which are considered an important source of PDGF in the development of pulmonary fibrosis, is upregulated by DEX [12].

Since excessive fibroblast proliferation is a central event in the pathogenesis of pulmonary fibrosis and may be driven by PDGF, it was investigated whether DEX treatment influenced the fibroblast mitogenic activity of BAL fluid from infants at risk of CLD. Additionally, the effect of DEX treatment on PDGF-BB levels in BAL fluid was examined. In order to analyse the effect of DEX treatment on pulmonary collagen synthesis, levels of N-terminal-propeptide of collagen type III (PIIINP) as a marker of collagen type III synthesis and pulmonary fibrosis were determined [13]. In order to determine the anti-inflammatory effect of DEX, interleukin (IL)-1 $\beta$ levels and BAL cell numbers were determined. 


\section{Methods}

\section{Patients and dexamethasone regimen}

Fifteen ventilated preterm infants with a gestational age of $<30$ weeks were included in the study. Criteria for inclusion were: 1) ventilator dependency because of RDS; and 2) treatment with DEX because of risk of CLD development. Informed consent from the parents and approval by the local medical ethics committees were obtained. All infants received $0.5 \mathrm{mg} \cdot \mathrm{kg}^{-1} \cdot \mathrm{day}^{-1} \mathrm{DEX}$ sodium phosphate, administered intravenously in two doses, for 3 days. This was followed by $0.3 \mathrm{mg} \cdot \mathrm{kg}^{-1} \cdot \mathrm{day}^{-1}$ in two divided doses for an additional 3 days, after which the infants were weaned from over 2-4 weeks. At the time that the DEX treatment was started, sputum cultures were negative for microorganisms. One patient had a Ureaplasma urealyticum-positive sputum culture 2 days prior to DEX treatment and was treated with erythromycin. In addition, none of the patients exhibited signs of active infection at the time the DEX treatment was initiated. CLD was defined as having an abnormal chest radiograph and oxygen dependency at 28 days of age [14].

\section{Bronchoalveolar lavage}

BAL was performed as described by GRIGG et al. [15], using two aliquots of $1 \mathrm{ml}$ saline $\cdot \mathrm{kg}$ body weight ${ }^{-1}$ within $24 \mathrm{~h}$ prior to initiating DEX treatment and $24-72 \mathrm{~h}$ after initiation of treatment or just before weaning from the ventilator, whichever occurred first. The BAL samples obtained were immediately put on ice and processed within $1 \mathrm{~h}$. The recovered volume was determined and thereafter the samples were centrifuged $\left(10 \mathrm{~min}\right.$ at $420 \times g$ at $4^{\circ} \mathrm{C}$ ). The fluid fraction was separated from the cellular fraction and the BAL fluid stored in aliquots at $-80^{\circ} \mathrm{C}$ until analysis. Cell numbers were determined using a haemocytometer. May-Grünwald Giemsa staining was performed on cytospin preparations and cell differentials were determined by counting 300 cells per cytospin.

\section{Fibroblast proliferation assay}

The human foetal lung fibroblast cell line HFL-1 was cultured in Dulbecco modified Eagle medium (DMEM) supplemented with $10 \%$ foetal calf serum (FCS), $4 \mathrm{mM}$ ultraglutamine-I (BioWhittaker Europe, Verviers, Belgium), antibiotics (penicillin, $100 \mathrm{U} \cdot \mathrm{mL}^{-1}$; streptomycin, $100 \mu \mathrm{g} \cdot \mathrm{mL}^{-1}$ ) and $15 \mathrm{mM}$ hydroxyethylpiperazine ethanesulphonic acid (HEPES) buffer. BAL fluid-induced fibroblast proliferation was determined using a colorimetric assay based on the uptake and subsequent release of methylene blue [16]. Fibroblasts were seeded $\left(6 \times 10^{3}\right.$ cells $\cdot$ well $\left.{ }^{-1}\right)$ into 96 -well microtitre plates in DMEM $/ 0.4 \%$ FCS and allowed to adhere for $24 \mathrm{~h}$. Owing to limited BAL fluid volumes, initial experiments were performed with serial dilutions of BAL fluid (1/16 to $1 / 1,024$ in DMEM $/ 0.4 \%$ FCS) from infants at risk of CLD. These experiments revealed that the mitogenic activity of the BAL fluid was maximal at $1 / 16$ dilution and decreased with increasing dilution down to medium control (DMEM/ $/ 4 \%$ FCS) levels (data not shown). Therefore, in further studies, a $1 / 16$ dilution of BAL fluid was used to determine the effect on fibroblast proliferation. Proliferation was determined in triplicate after $48 \mathrm{~h}$ and expressed as percentage change in mean absorbance from that of cells exposed to DMEM $/ 0.4 \%$ FCS alone. The assay was validated by direct cell counting and tritium thymidine incorporation.

\section{Analysis of bronchoalveolar lavage fluid}

PDGF-BB levels were determined using an enzyme-linked immunosorbent assay (ELISA; R\&D Systems, Abingdon, UK), PIIINP levels using a radioimmunoassay (Orion diagnostica, Espoo, Finland) and IL-1 $\beta$ levels by ELISA (Human IL-1 $\beta$ cytoset $^{\text {TM; }}$ Biosource International, Camarillo, CA, USA). Assays were performed according to the methodology of the manufacturers. The detection limits were $4.6 \mathrm{pg} \cdot \mathrm{mL}^{-1}, 0.2 \mu \mathrm{g} \cdot \mathrm{L}^{-1}$ and $1.6 \mathrm{pg} \cdot \mathrm{mL}^{-1}$, respectively. It was previously demonstrated that when BAL fluid is analysed in this way, there is a strong correlation between parameters determined in BAL fluid and the corrected concentrations of these parameters (i.e. expressed per millilitre of epithelial lining fluid) [17]. Therefore, no marker for dilution was used in the present study, and PDGF-BB, PIIINP and IL-1 $\beta$ levels in BAL fluid are presented as volume concentrations, which is also in accordance with the most recent European Respiratory Society task force guidelines on BAL in children [18].

\section{Inhibition of bronchoalveolar lavage fluid-induced fibroblast proliferation}

Tyrphostin AG1296 is a compound that specifically inhibits PDGF receptor tyrosine kinase, thereby inhibiting PDGFinduced proliferation [19]. After adherence of the cells for $24 \mathrm{~h}$, the medium was changed to DMEM alone for $6 \mathrm{~h}$. Thereafter, fresh DMEM containing $30 \mu \mathrm{M}$ tyrphostin AG1296 (Calbiochem, Darmstadt, Germany) in vehicle (dimethylsulphoxide) or vehicle alone was added for a further $18 \mathrm{~h}$. The medium was replaced by BAL fluid (known to contain PDGF-BB) in DMEM $/ 0.4 \%$ FCS for $48 \mathrm{~h}$. The assay was performed in triplicate. As control, HFL-1 cells preincubated with AG1296 or vehicle were stimulated with $50 \mathrm{ng} \cdot \mathrm{mL}^{-1}$ PDGF-BB (R\&D Systems).

\section{Statistical analysis}

Patient characteristics and BAL fluid cell numbers are presented as mean \pm SEM. Fibroblast proliferation results and PDGF-BB, PIIINP and IL-1 $\beta$ levels are presented as median (range). A paired t-test was used for comparison of values obtained before and after initiation of DEX treatment. Pearson's correlation was used to compare the relationship between BAL fluid mitogenic activity and PDGF-BB concentrations. A p-value of $<0.05$ was considered significant.

\section{Results}

\section{Patients and dexamethasone regimen}

The patients $(n=15)$ had a gestational age of $27.2 \pm 0.3$ weeks (range 26-30 weeks) and a birthweight of 972.6 $\pm 66.2 \mathrm{~g}$ (range $650-1,529 \mathrm{~g})$. All infants were intubated and ventilated immediately after birth and all were still ventilator-dependent at the time that DEX treatment was started. DEX treatment was initiated at an age of $18.3 \pm 2$ days (range 7-33 days) after birth. In response to the DEX therapy, the pulmonary function of the infants improved and consequently they were extubated within days after initiation of treatment. Despite treatment, all patients developed CLD. One of the infants died, although this was not during the study period. The overall survival of the studied infants was $93.3 \%$. 


\section{Bronchoalveolar lavage}

BAL was performed at $2.3 \pm 0.2$ days (range $1-3$ days) after initiation of DEX treatment. BAL fluid recovery was determined before and after initiation of treatment. No difference was observed in BAL fluid recovery before and after initiation of DEX $(40.1 \pm 5.2$ and $33.9 \pm 4.2 \%$ of initial lavage volume, respectively). Table 1 shows the numbers and relative composition of the BAL fluid population. The total number of cells as well as the number of cells per millilitre of BAL fluid was significantly decreased after DEX treatment. The percentages of neutrophils and macrophages in the cell population were not influenced by DEX treatment. However, the absolute numbers of macrophages per millilitre of BAL fluid decreased significantly after DEX. The number of neutrophils per millilitre of BAL fluid also declined after DEX, but did not reach significance.

\section{Bronchoalveolar lavage fluid-induced fibroblast proliferation}

For the whole group of patients, BAL fluid-induced fibroblast proliferation increased from $34.7 \%(7.7-75.3 \%)$ before DEX to $51.2 \%(17.8-115.1 \%)$ after initiation of DEX treatment $(\mathrm{p}<0.05)$. Of the 15 patients studied, 12 showed an increase and three a decrease in BAL fluid-induced fibroblast proliferation after initiation of DEX treatment (fig. 1a). Interestingly, the three patients with decreased BAL fluid mitogenicity showed the highest mitogenic activity before initiation of DEX. When fibroblast proliferation was expressed relative to 5\% FCS, similar results were obtained (data not shown).

\section{Analysis of bronchoalveolar lavage fluid}

A summary of the analysis of the individual BAL fluid samples is presented in table 2 .

DEX treatment did not influence PDGF-BB concentration in the BAL fluid from the 15 patients before and after DEX (11.8 $(<4.6-92.9)$ versus $27(<4.6-80.4) \mathrm{pg} \cdot \mathrm{mL} \mathrm{BAL} \mathrm{fluid}{ }^{-1}$, respectively). However, when the mitogenic activity of BAL fluid from patients decreased after DEX, this decrease was accompanied by a decrease in PDGF-BB levels $(n=3)$. Of the 12 patients with increased mitogenic activity after DEX, 10 showed an increase in PDGF-BB levels after DEX and one a minimal decrease; one had undetectable PDGF-BB levels before and after DEX (fig. 1b). A significant positive correlation was observed between the change in PDGF-BB levels in BAL fluid and the change in BAL fluid-induced fibroblast proliferation after initiation of DEX treatment $(\mathrm{r}=0.7 ; \mathrm{p}<0.01)$.

PIIINP levels were determined in BAL fluid from 10 patients (based on sample availability). DEX treatment did not significantly change the levels of PIIINP in this group of 10 patients $\left(10.5(7.6-51.2) \mu \mathrm{g} \cdot \mathrm{L}\right.$ BAL fluid ${ }^{-1}$ before DEX versus $11.5(6-215) \mu \mathrm{g} \cdot \mathrm{L}$ BAL fluid ${ }^{-1}$ after DEX.

Table 1.-Effect of dexamethasone (DEX) treatment on the cell population in neonatal bronchoalveolar lavage fluid

\begin{tabular}{lcc}
\hline & Before DEX & After DEX \\
\hline Total cells $10^{6}$ cells & $1.39 \pm 0.31$ & $0.51 \pm 0.07^{*}$ \\
Total cells $10^{6}$ cells $\cdot \mathrm{mL}^{-1}$ & $1.74 \pm 0.39$ & $0.9 \pm 0.26^{*}$ \\
Macrophages \% & $43.7 \pm 5.8$ & $39.8 \pm 6.4$ \\
Neutrophils \% & $48.6 \pm 6.0$ & $47.3 \pm 7.0$ \\
Macrophages $10^{6}$ cells $\cdot \mathrm{mL}^{-1}$ & $0.7 \pm 0.16$ & $0.28 \pm 0.06^{*}$ \\
Neutrophils $10^{6}$ cells $\cdot \mathrm{mL}^{-1}$ & $0.93 \pm 0.27$ & $0.54 \pm 0.23$ \\
\hline
\end{tabular}

Data are presented as mean \pm SEM. $*: p<0.05$.
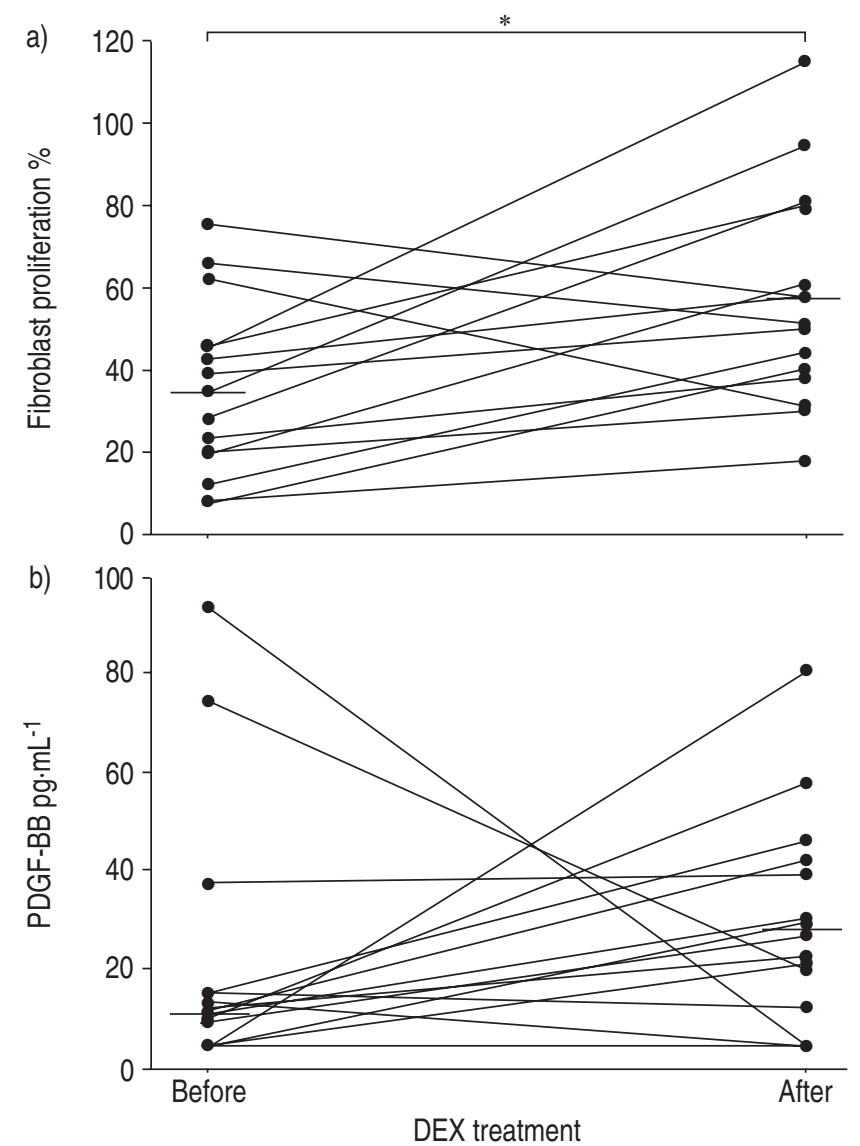

Fig. 1.-a) Effect of bronchoalveolar lavage (BAL) fluid (1/16 dilution) on fibroblast proliferation. b) Platelet-derived growth factor (PDGF)-BB concentration before and after dexamethasone (DEX) treatment for each individual. Horizontal bars represent medians. Twelve patients show an increase in BAL fluid mitogenic activity after DEX and three a decrease. The three patients with a decrease in BAL fluid mitogenic activity after DEX also show a decrease in PDGF-BB levels after DEX. Of the 12 patients with increased mitogenic activity, 10 show increased PDGF-BB levels after DEX and one a minimal decline in PDGF-BB level after DEX; in one patient, PDGF-BB was undetectable before and after DEX treatment and so is shown as being at the detection limit of the assay. *: $\mathrm{p}<0.05$.

Owing to limited amounts of BAL fluid, IL-1 $\beta$ levels were investigated in eight patients. A significant decrease in IL-1 $\beta$ levels in BAL fluid was observed after DEX treatment $(31.3$ $(<1.6-78.8) \mathrm{pg} \cdot \mathrm{mL}$ BAL fluid $^{-1}$ before DEX versus $<1.6$ $(<1.6-68.2) \mathrm{pg} \cdot \mathrm{mL}$ BAL fluid ${ }^{-1}$ after DEX; $\left.<<0.05\right)$. Seven patients showed decreased IL-1 $\beta$ levels after compared to before DEX treatment. In one patient, IL-1 $\beta$ was undetectable before and after DEX treatment.

\section{Inhibition of bronchoalveolar lavage fluid-induced fibroblast proliferation}

In order to determine whether PDGF-BB contributed to BAL fluid mitogenicity, the PDGF receptor system in fibroblasts was blocked using the specific inhibitor tyrphostin AG1296. Tyrphostin AG1296 was able to inhibit both BAL fluid- and PDGF-BB-induced fibroblast proliferation (fig. 2). Tyrphostin AG1296 had no effect on HFL-1 cells after $48 \mathrm{~h}$ in DMEM containing $0.4 \%$ FCS compared to preincubation with vehicle alone. 
Table 2.-Analysis of bronchoalveolar lavage fluid of individual patients before and after dexamethasone treatment

\begin{tabular}{|c|c|c|c|c|c|c|c|c|}
\hline \multirow[t]{2}{*}{ Patient no. } & \multicolumn{2}{|c|}{ Fibroblast proliferation $\%$} & \multicolumn{2}{|c|}{ PDGF-BB pg $\cdot \mathrm{mL}^{-1}$} & \multicolumn{2}{|c|}{ PIIINP $\mu \mathrm{g} \cdot \mathrm{L}^{-1}$} & \multicolumn{2}{|c|}{$\mathrm{IL}-1 \beta \mathrm{pg} \cdot \mathrm{mL}^{-1}$} \\
\hline & Before & After & Before & After & Before & After & Before & After \\
\hline 1 & 38.9 & 50.0 & 15.2 & 46.1 & ND & ND & 52.3 & 26.1 \\
\hline 2 & 23.3 & 38.3 & 15.1 & 12.3 & ND & ND & ND & ND \\
\hline 3 & 42.6 & 58.1 & 11.8 & 42.1 & 7.7 & 16.2 & ND & ND \\
\hline 4 & 45.9 & 115.1 & $<4.6$ & 29.7 & ND & ND & ND & ND \\
\hline 5 & 7.7 & 40.3 & $<4.6$ & 80.4 & 7.6 & 215 & 78.8 & 68.2 \\
\hline 6 & 19.5 & 60.8 & $<4.6$ & 20.9 & 51.2 & 18.2 & $<1.6$ & $<1.6$ \\
\hline 7 & 20.5 & 30.2 & 37.1 & 39.1 & 43.1 & 25.9 & 23.2 & 5.3 \\
\hline 8 & 34.7 & 94.4 & 11.0 & 30.4 & 12.3 & 23.2 & ND & ND \\
\hline 9 & 46.0 & 79.2 & 12.6 & 22.8 & 9.5 & 6.6 & 30.7 & $<1.6$ \\
\hline 10 & 28.3 & 80.8 & 9.3 & 27.0 & ND & ND & 48.9 & $<1.6$ \\
\hline 11 & 8.3 & 17.8 & $<4.6$ & $<4.6$ & 9.5 & 6.6 & ND & ND \\
\hline 12 & 12.1 & 44.2 & 10.5 & 57.5 & ND & ND & ND & ND \\
\hline 13 & 66.2 & 51.2 & 74.0 & 19.7 & 9.5 & 6.8 & 31.8 & $<1.6$ \\
\hline 14 & 75.3 & 57.6 & 13.6 & $<4.6$ & 24.5 & 6.0 & 30.7 & $<1.6$ \\
\hline 15 & 62.2 & 31.3 & 92.8 & $<4.6$ & 11.4 & 6.3 & ND & ND \\
\hline Median & 34.7 & $51.2^{*}$ & 11.8 & 27.0 & 10.5 & 11.5 & 31.3 & $<1.6^{*}$ \\
\hline Range & $7.7-75.3$ & $17.8-115.1$ & $<4.6-92.8$ & $<4.6-80.4$ & $7.6-51.2$ & $6.0-215$ & $<1.6-78.8$ & $<1.6-68.2$ \\
\hline
\end{tabular}

PDGF: platelet-derived growth factor; PIINP: N-terminal propeptide of collagen type III; IL: interleukin; ND: not determined. *: $\mathrm{p}<0.05$ versus before dexamethasone.

\section{Discussion}

Anti-inflammatory therapy with the corticosteroid DEX is commonly used in neonatal intensive care units to treat prematurely born infants with or at risk of CLD. This treatment has been proven to be beneficial in the short term as it improves pulmonary function, facilitates weaning from the ventilator and rapidly reduces pulmonary inflammation [8]. Conversely, there are many side-effects associated with DEX treatment, such as growth retardation, hyperglycaemia, hypertension, myocardial hypertrophy, gastrointestinal perforation and neurological impairment $[20,21]$. Furthermore, it is not known whether DEX treatment affects the development of pulmonary fibrosis in CLD, which may impair pulmonary function in the long term.

In the present study, it was demonstrated that BAL fluid from infants developing CLD contains mitogenic activity for human foetal lung fibroblasts. Recently, CURRIE et al. [22]

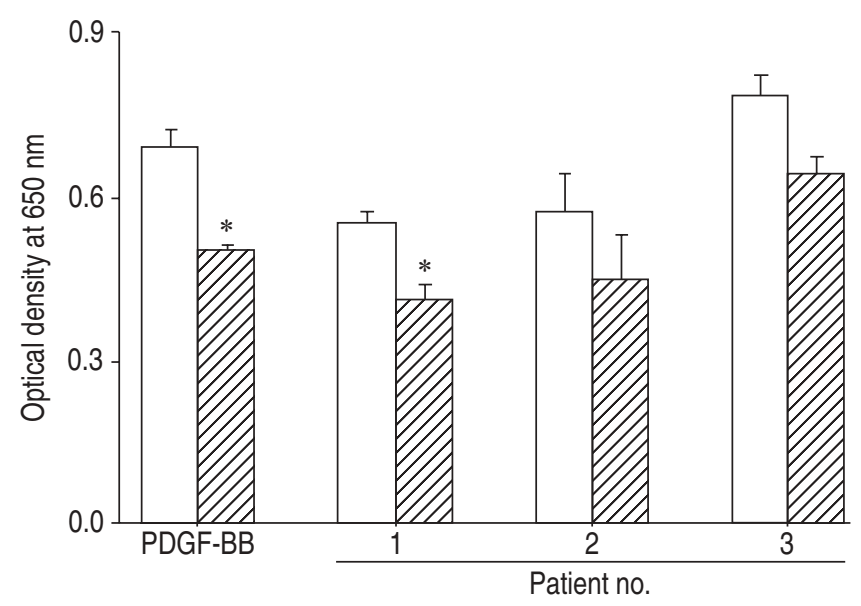

Fig. 2. - Effect of tyrphostin AG1296 (ש्Z) on platelet-derived growth factor (PDGF)-BB (50 ng. $\left.\mathrm{mL}^{-1}\right)$ - and bronchoalveolar lavage (BAL) fluid-induced fibroblast proliferation as indicated by change in optical density after methylene blue elution ( $\square$ : vehicle alone). Data are presented as mean \pm SEM. Results for three individual patients are shown. *: $\mathrm{p}<0.05$. demonstrated that BAL fluid from infants developing CLD is mitogenic for fibroblasts. However, in that study, mice embryonic fibroblasts were used. The present study is of importance since human foetal lung fibroblasts were used and mitogenic activity was also detected in BAL fluid. This strengthens the findings reported by CURRIE et al. [22], especially since cells from different species may respond differently to certain stimuli. A novel finding of the present study is that treating infants who are developing CLD with systemic DEX does not reduce BAL fluid mitogenicity for human foetal lung fibroblasts. This finding is important in the context of the development of pulmonary fibrosis as excessive fibroblast proliferation is one of the mechanisms involved in fibrosis development [4, 10]. Therefore, the present data do not support the use of DEX to prevent pulmonary fibrosis in patients at risk of CLD.

In accordance with previous studies [8, 23], DEX treatment reduced the level of the pro-inflammatory cytokine IL-1 $\beta$ and inflammatory cell numbers in the BAL samples. It is likely that this reduction in inflammation contributed to the observed improvement in pulmonary function in all infants and facilitated weaning from the ventilator. However, the present study indicates that, although DEX treatment reduces pulmonary inflammation, it does not reduce pulmonary fibroproliferation in infants who develop CLD. From the present data, it can even be speculated that DEX treatment enhances the fibroproliferative response in the lungs of these infants. In this sense, DEX therapy would have two separate and dissociated effects: first, reducing inflammation, but secondly, enhancing fibrosis.

DEX itself may also influence the proliferative activity of fibroblasts in a direct way. It has been reported that DEX stimulates the proliferation of rat lung fibroblasts in vitro, presumably by increasing the expression of the PDGF- $\alpha$ receptor [24]. In vitro studies with DEX in primary human foetal lung fibroblasts, however, revealed reduced proliferation [25]. The BAL samples obtained after treatment in the present study may have contained DEX as a result of pulmonary vascular leakage, which is clearly associated with CLD development [3]. Therefore, if DEX were present in the analysed BAL fluid samples, it may have influenced the BAL fluid-induced fibroblast proliferation. However, in the present culture system, DEX itself was unable to stimulate fibroblast 
proliferation. Furthermore, addition of the glucocorticoid antagonist RU 38486 to BAL fluid obtained after DEX treatment did not influence BAL fluid-induced fibroblast proliferation and neither did the addition of DEX to BAL samples obtained before DEX treatment (data not shown). Therefore, it is unlikely that, if DEX were present in BAL fluid, it influenced the observed BAL fluid-induced fibroproliferation.

Animal models of acute lung injury and pulmonary fibrosis reveal increased expression of PDGF-B mRNA and PDGF$\mathrm{BB}$ prior to deoxyribonucleic acid synthesis and tissue repair $[11,26,27]$. In addition, PDGF-BB has been shown to contribute to the fibroblast mitogenic activity of BAL fluid in bleomycin-induced pulmonary fibrosis [11]. Furthermore, inhibition of PDGF-BB reduces bleomycin-induced pulmonary fibrosis in mice [28]. In line with this is the correlation found in the present study between the change in BAL fluidinduced fibroblast proliferation and the concomitant change in PDGF-BB levels before and after DEX treatment. This suggests that the increase in PDGF-BB is at least partly responsible for the observed increase in BAL fluid-induced fibroblast proliferation after DEX treatment. Blocking the PDGF receptor system in fibroblasts resulted in a reduction in BAL fluid-induced fibroblast proliferation. Although this indicates that PDGF may be an important fibroblast mitogen in the pathophysiology of CLD, other fibroblast mitogens are also likely to be involved, since blocking the PDGF receptor system did not always result in a significant reduction in BAL fluid-induced fibroblast proliferation.

Alveolar macrophages are considered a major source of PDGF in pulmonary fibrosis and increased numbers are present in the pathogenesis of CLD [2, 4]. In the present study, the BAL cell population contained considerable numbers of alveolar macrophages. Therefore, it is likely that these cells are sources of the PDGF detected in the BAL fluid. It has been demonstrated that macrophages stimulated with DEX express increased amounts of PDGF-B mRNA, secrete increased amounts of PDGF and stimulate fibroblast proliferation and collagen synthesis [12, 29]. Therefore, it may well be that systemic DEX treatment resulted in an upregulation of PDGF-BB production and secretion by alveolar macrophages. Activation of such a pathway may result in a profibrotic environment in the lungs of infants with or at risk of CLD.

In vitro experiments have demonstrated that DEX is able to reduce collagen synthesis by fibroblasts [30, 31]. In line with this, DEX treatment has been demonstrated to decrease total body collagen synthesis in infants with CLD [9, 32]. However, no difference was observed in PIIINP levels before and after DEX treatment, indicating that DEX treatment does not influence collagen synthesis in the lungs of infants developing CLD, and, therefore, may not inhibit the development of pulmonary fibrosis in these infants. This is in agreement with a recent study showing that DEX treatment does not inhibit but rather stimulates collagen synthesis by human foetal lung fibroblasts [25]. Interestingly, CHEN et al. [33] demonstrated that prenatal DEX administration with prolonged exposure of preterm rats to hyperoxia resulted in a pulmonary pathological picture similar to CLD and with even greater severity of septal fibrosis compared to hyperoxia-exposed control rats. This implies that DEX treatment does not necessarily inhibit pulmonary collagen synthesis in vivo. However, the fact that no effect of DEX treatment on PIIINP levels in BAL fluid was observed may be due to the limited number of patients and great variability in PIIINP levels, as has also been shown to exist in BAL fluid from patients with ARDS [10, 34]. Alternatively, an effect of DEX on PIIINP levels may be obscured due to drainage of PIIINP from the lung by lymph vessels [35].

In the present study, three patients with relatively high
BAL fluid mitogenicity before initiation of DEX treatment showed a decrease in BAL fluid mitogenicity after DEX. In contrast, 12 patients with relatively low BAL fluid mitogenicity before DEX showed an increase after DEX. The observed changes in BAL fluid mitogenicity and PDGF-BB levels after DEX treatment did not correlate with surfactant treatment, antenatal steroids, type of ventilation, birthweight or gestational age (data not shown). Although suggestive, it cannot be concluded that two different types of response to DEX treatment occur in infants developing CLD as only a small group of patients were studied, pulmonary function tests were not performed and long-term pulmonary outcome was not examined. Studies including a larger number of patients may address this question.

It cannot be excluded that the observed differences in the determined parameters in BAL fluid before and after DEX treatment simply reflect the natural course of the disease, since no control patients were included in this study. Because of the variation in initiation of DEX treatment in the present patient population (7-33 days after birth) and the fact that both centres find it unethical to withdraw patients at risk of CLD from DEX treatment, a placebo control group was not included. However, regarding the expected and observed antiinflammatory effects of DEX treatment and the heterogeneity of the postnatal ages at which DEX treatment was initiated, it is tempting to speculate that the effect on BAL fluid mitogenicity and PDGF-BB levels is likely to be due to DEX treatment.

In conclusion, the present study suggests that systemic dexamethasone treatment, as opposed to its anti-inflammatory action, does not reduce pulmonary fibroproliferation in chronic lung disease of prematurity. Therefore, this study does not support the use of dexamethasone for preventing the fibrotic response in infants at risk of chronic lung disease of prematurity. Taking the present finding and the already known complications into account, the present authors believe that treatment with dexamethasone for the prevention of chronic lung disease of prematurity should be used with caution.

\footnotetext{
Acknowledgements. The authors would like to thank R. Benner and V.H.J. van der Velden for their critical reading of the manuscript and M. Huysman for assistance with bronchoalveolar lavage.
}

\section{References}

1. Merritt TA, Cochrane CG, Holcomb K, et al. Elastase and alpha 1-proteinase inhibitor activity in tracheal aspirates during respiratory distress syndrome. Role of inflammation in the pathogenesis of bronchopulmonary dysplasia. J Clin Invest 1983; 72: 656-666.

2. Ogden BE, Murphy SA, Saunders GC, Pathak D, Johnson JD. Neonatal lung neutrophils and elastase/proteinase inhibitor imbalance. Am Rev Respir Dis 1984; 130: 817-821.

3. Pierce MR, Bancalari E. The role of inflammation in the pathogenesis of bronchopulmonary dysplasia. Pediatr Pulmonol 1995; 19: 371-378.

4. McAnulty RJ, Laurent GJ. Pathogenesis of lung fibrosis and potential new therapeutic strategies. Exp Nephrol 1995; 3: 96-107.

5. Cherukupalli K, Larson JE, Rotschild A, Thurlbeck WM. Biochemical, clinical, and morphologic studies on lungs of infants with bronchopulmonary dysplasia. Pediatr Pulmonol 1996; 22: 215-229.

6. Gerdes JS, Harris MC, Polin RA. Effects of dexamethasone and indomethacin on elastase, alpha 1-proteinase inhibitor, 
and fibronectin in bronchoalveolar lavage fluid from neonates. J Pediatr 1988; 113: 727-731.

7. Kotecha S, Wangoo A, Silverman M, Shaw RJ. Increase in the concentration of transforming growth factor beta-1 in bronchoalveolar lavage fluid before development of chronic lung disease of prematurity. J Pediatr 1996; 128: 464469.

8. Yoder MC Jr, Chua R, Tepper R. Effect of dexamethasone on pulmonary inflammation and pulmonary function of ventilator-dependent infants with bronchopulmonary dysplasia. Am Rev Respir Dis 1991; 143: 1044-1048.

9. Co E, Chari G, McCulloch K, Vidyasagar D. Dexamethasone treatment suppresses collagen synthesis in infants with bronchopulmonary dysplasia. Pediatr Pulmonol 1993; 16: 36-40.

10. Marshall RP, Bellingan G, Webb S, et al. Fibroproliferation occurs early in the acute respiratory distress syndrome and impacts on outcome. Am J Respir Crit Care Med 2000; 162: 1783-1788.

11. Walsh J, Absher M, Kelley J. Variable expression of plateletderived growth factor family proteins in acute lung injury. Am J Respir Cell Mol Biol 1993; 9: 637-644.

12. Haynes AR, Shaw RJ. Dexamethasone-induced increase in platelet-derived growth factor (B) mRNA in human alveolar macrophages and myelomonocytic HL60 macrophage-like cells. Am J Respir Cell Mol Biol 1992; 7: 198-206.

13. Bjermer L, Lundgren R, Hallgren R. Hyaluronan and type III procollagen peptide concentrations in bronchoalveolar lavage fluid in idiopathic pulmonary fibrosis. Thorax 1989; 44: 126-131.

14. Bancalari E, Abdenour GE, Feller R, Gannon J. Bronchopulmonary dysplasia: clinical presentation. J Pediatr 1979; 95: 819-823.

15. Grigg J, Arnon S, Silverman M. Fractional processing of sequential bronchoalveolar lavage fluid from intubated babies. Eur Respir J 1992; 5: 727-732.

16. Oliver MH, Harrison NK, Bishop JE, Cole PJ, Laurent GJ. A rapid and convenient assay for counting cells cultured in microwell plates: application for assessment of growth factors. J Cell Sci 1989; 92: 513-518.

17. IJsselstijn H, Zimmermann LJ, Bunt JE, de Jongste JC, Tibboel D. Prospective evaluation of surfactant composition in bronchoalveolar lavage fluid of infants with congenital diaphragmatic hernia and of age-matched controls. Crit Care ed 1998; 26: 573-580.

18. de Blic J, Midulla F, Barbato A, et al. Bronchoalveolar lavage in children. Eur Respir J 2000; 15: 217-231.

19. Rice AB, Moomaw CR, Morgan DL, Bonner JC. Specific inhibitors of platelet-derived growth factor or epidermal growth factor receptor tyrosine kinase reduce pulmonary fibrosis in rats. Am J Pathol 1999; 155: 213-221.

20. Barrington KJ, Finer NN. Treatment of bronchopulmonary dysplasia. A review. Clin Perinatol 1998; 25: 177-202.

21. Barrington KJ. The adverse neuro-developmental effects of postnatal steroids in the preterm infant: a systematic review of RCTs. BMC Pediatr 2001; 1: 1-9.

22. Currie AE, Kelly M, Vyas JR, Pandya H, Field D, Kotecha S.
Fibroblast mitogenic activity of lung lavage fluid from infants with chronic lung disease of prematurity. Arch Dis Child Fetal Neonatal Ed 2002; 86: F193-F197.

23. Groneck P, Reuss D, Gotze-Speer B, Speer CP. Effects of dexamethasone on chemotactic activity and inflammatory mediators in tracheobronchial aspirates of preterm infants at risk for chronic lung disease. J Pediatr 1993; 122: 938-944.

24. Warshamana GS, Martinez S, Lasky JA, Corti M, Brody AR. Dexamethasone activates expression of the PDGF-alpha receptor and induces lung fibroblast proliferation. Am J Physiol 1998; 274: L499-L507.

25. Brenner RE, Felger D, Winter C, Christiansen A, Hofmann D, Bartmann P. Effects of dexamethasone on proliferation, chemotaxis, collagen I, and fibronectin-metabolism of human fetal lung fibroblasts. Pediatr Pulmonol 2001; 32: 1-7.

26. Fabisiak JP, Evans JN, Kelley J. Increased expression of PDGF-B (c-sis) mRNA in rat lung precedes DNA synthesis and tissue repair during chronic hyperoxia. Am J Respir Cell Mol Biol 1989; 1: 181-189.

27. Han RN, Buch S, Freeman BA, Post M, Tanswell AK. Platelet-derived growth factor and growth-related genes in rat lung. II. Effect of exposure to $85 \% \mathrm{O}_{2}$. Am J Physiol 1992 262: L140-L146.

28. Yoshida $\mathrm{M}$, Sakuma-Mochizuki J, Abe $\mathrm{K}$, et al. In vivo gene transfer of an extracellular domain of platelet-derived growth factor beta receptor by the HVJ-liposome method ameliorates bleomycin-induced pulmonary fibrosis. Biochem Biophys Res Commun 1999; 265: 503-508.

29. Song E, Ouyang N, Horbelt M, Antus B, Wang M, Exton MS. Influence of alternatively and classically activated macrophages on fibrogenic activities of human fibroblasts. Cell Immunol 2000; 204: 19-28.

30. Sterling KM Jr, Harris MJ, Mitchell JJ, DiPetrillo TA, Delaney GL, Cutroneo KR. Dexamethasone decreases the amounts of type I procollagen mRNAs in vivo and in fibroblast cell cultures. J Biol Chem 1983; 258: 7644-7647.

31. Cockayne D, Sterling KM Jr, Shull S, Mintz KP, Illeyne S, Cutroneo KR. Glucocorticoids decrease the synthesis of type I procollagen mRNAs. Biochemistry 1986; 25: 3202-3209.

32. Crofton PM, Shrivastava A, Wade JC, et al. Effects of dexamethasone treatment on bone and collagen turnover in preterm infants with chronic lung disease. Pediatr Res 2000; 48: $155-162$.

33. Chen Y, Martinez MA, Frank L. Prenatal dexamethasone administration to premature rats exposed to prolonged hyperoxia: a new rat model of pulmonary fibrosis (bronchopulmonary dysplasia). J Pediatr 1997; 130: 409-416.

34. Clark JG, Milberg JA, Steinberg KP, Hudson LD. Type III procollagen peptide in the adult respiratory distress syndrome. Association of increased peptide levels in bronchoalveolar lavage fluid with increased risk for death. Ann Intern Med 1995; 122: 17-23.

35. Harrison NK, McAnulty RJ, Kimpton WG, Fraser JR, Laurent TC, Laurent GJ. Heterogeneity of type III procollagen N-terminal peptides in BAL fluid from normal and fibrotic lungs. Eur Respir J 1993; 6: 1443-1448. 